\title{
Compact four-dimensional euclidean space embedded in the universe
}

\begin{abstract}
A compact 4-dimensional manifold whose metric tensor has a positive determinant (named "Euclid ball") is considered. The Euclid ball can be embedded in the Lorentzian space, and it exists stably through the history of the universe. Since the Euclid ball has the same metric as that of the Schwarzschild black hole on its 3-dimensional surface, asymptotic observers cannot distinguish them. Euclid balls can be created in the universe before the cosmological inflation (pre-universe). If large fraction of whole energy of the pre-universe was encapsulated in Euclid balls, they behave as the dark matter in the current universe. If Euclid balls formed at the end of the inflation with a heavy mass, they can be a seed of supper-massive black-holes, which are necessary to initiate a forming of galaxies in the early universe. The primordial gravitational wave and/or -ray bursts at early universe are a possible signal of the Euclidean ball.
\end{abstract}

Keywords: compact 4-dimensional manifold, euclid ball, lorentzian space, schwarzschild black hole, supper-massive black-holes
Volume 2 Issue 3 - 2018

\author{
Yoshimasa Kurihara \\ The High Energy Accelerator Organization (KEK), Japan
}

Correspondence: Yoshimasa Kurihara,The High Energy Accelerator Organization (KEK), Oho I-I,Tsukuba, Ibaraki 305-080I, Japan, Tel +8I29 8796-088,

EmailYoshimasa.kurihara@kek.jp

Received: June 06, 2018 | Published: June 19, 2018

\section{Introduction}

The dark matter (DM) is, at first, introduced to explain an anomaly of stellar velocities in the galaxy. ${ }^{1}$ The existence of the DM is now widely accepted ${ }^{2}$ due to large number of observational supports. ${ }^{3,4}$ The DM is also necessary to explain galaxy formations in early universe. ${ }^{5}$ A 3-dimensional map of the DM distribution is made using a gravitational lensing effect. ${ }^{6}$ On the other hand, a direct observation of the DM is still not succeeded though number of experimental searches of the DM has been going on. Even though the cold DM density of the universe is precisely measured ${ }^{7,8}$ as $\Omega_{\mathrm{CDM}}=0.258(11)$ , still it is not identified what it is experimentally. Several candidates of the cold DM are proposed, such as the lightest particle in the SUSY model (LSP), axions ${ }^{9,10}$ introduced to solve the strong $C P$ problem, primordial black holes ${ }^{11}$ and so on. Though recent direct searches of the SUSY particles at the LHC experiments report negative results, still a weak interacting LSP can be the DM candidate. Astroparticle discussions give additional constraint ${ }^{12,13}$ for the SUSY model to avoid the overproduction of the SUSY-DM. Each scenario has its advantages and disadvantages, and is not conclusive yet. In this report, we propose new candidate of the DM based on the geometrical model including quantum effects of the general relativity.

\section{Euclid-ball solution}

A 4-dimensional smooth manifold can be categorised by a sign of a determinant of their metric tensor. It is referred to as a metric-sign hereafter. If a manifold is diffeomorphism to the Euclid space, whose metric tensor is $-\zeta_{\text {.. }}=\operatorname{diag}(1,1,1,1)$, it is called the Euclidean-space in this report, even if it is highly curved space. If the Eulidean-space is a compact, it is referred to as the Euclid-ball (EB). The smooth manifold, which is diffeomorphism to a space with a metric tensor $\eta_{\text {.. }}=\operatorname{diag}(1,-1,-1,-1)$, is referred to as the Lorentzian space, again even if it is highly curved. Since a local space-time manifold without gravity is the (flat) Minkovski space and the general relativistic theory requires laws of physics must be diffeomophism, any space-time manifold in the universe is considered to be the Lorentzian space.
It is follows from a fact that the metric-sign is the diffeomorphism invariance. While the Euclid ball cannot be created during the entire history of the universe after the inflation, there is a possibility that EBs were created before the inflation, due to a stochastic effect of the preuniverse. ${ }^{14}$ An example of a EB-solution is a space with a metric of;

$$
-d s_{\mathrm{E}}^{2}=f_{\mathrm{E}}^{2}(r) d t^{2}+f_{\mathrm{E}}^{-2}(r) d r^{2}+r^{2} d \Omega
$$

At $r<r_{c}$, where $f_{\mathrm{E}}^{2}(r)=r_{c} / r-1$ and $d \Omega=d \theta^{2}+\sin ^{2} \phi d \phi^{2}$. The constant $r_{c}$ can be understood as the radius of the EB. One can confirm that scaler-and Ricci-curvature of this metric is zero. Therefore the EB metric is a solution of the Einstein equation in the Euclidean space with neither cosmological constant nor energy momentum tensor. Note that both of the Lorentzian and Euclidean spaces can be a solution of the Einstein equation with the null energy momentum tensor. The metric-sign is fixed by the energy momentum tensor and/ or the boundary condition. For cosmological solutions, one usually requires the energy momentum tensor with Lorentzian metric and/ or the asymptotic Minkovski space as the boundary condition. For instance, the Friedmann-Lmeître-Robertson-Walker (FLRW) metric ${ }^{1}$ with a positive curvature can be considered as a 3-dimensional sphere embedded in a 4-dimensional (flat) Euclid space. A region $r<1 / K^{1 / 2}$ is the Lorentzian-space, where $K$ is a curvature of the space.

The EB solution can be embedded in the Lorentzian-space by a following simple surgery: Preparing the Lorentzian-space with a Schwarzschild black hole whose metric as;

$$
d s_{\mathrm{M}}^{2}=f_{\mathrm{M}}^{2}(r) d t^{2}-f_{\mathrm{M}}^{-2}(r) d r^{2}-r^{2} d \Omega
$$

where $f_{\mathrm{M}}^{2}=1-r_{c} / r$. The solution in a region $r<r_{c}$ is replaced by the $\mathrm{EB}$, and adjust the angular coordinate $\theta$ and $\phi$ at the surface $r=r_{c}$. Two solutions give an identical metric at $r=r_{c}$ as $d s_{\mathrm{E}}^{2}\left(r=r_{c}\right) \stackrel{c}{=} d s_{\mathrm{M}}^{2}\left(r=r_{c}\right)$. The metric tensor is continuous (but not smooth) at the surface.

${ }^{1}$ see, for instance, ${ }^{15}$ 
There is no singularity at the event horizon of the Schwarzschild black hole as know well. This fact can be seen if we look at the metric using (modified) Kruskal-Szekeres coordinates. In the region $r>r_{c}$, a coordinate is taken as;

$$
\begin{aligned}
& U_{\mathrm{M}}=\left(\frac{r}{r_{c}}-1\right)^{1 / 2} e^{r / 2 r_{c}} \sinh \left(\frac{t}{2 r_{c}}\right), \\
& V_{\mathrm{M}}=\left(\frac{r}{r_{c}}-1\right)^{1 / 2} e^{r / 2 r_{c}} \cosh \left(\frac{t}{2 r_{c}}\right),
\end{aligned}
$$

which gives a line element as;

$$
d s_{\mathrm{M}}^{2}=4 \frac{r_{c}^{3}}{r} e^{-r / r_{c}}\left(d U_{\mathrm{M}}^{2}-d V_{\mathrm{M}}^{2}\right)-r^{2} d \Omega
$$

In this coordinate, $r$ can be written as a function of $U$ and $V$. In the region $r>r_{c}$, the Kruskal-Szekeres coordinates are modified as;

$$
U_{\mathrm{E}}=\left(1-\frac{r}{r_{c}}\right)^{1 / 2} e^{r / 2 r_{c}} \sin \left(\frac{\tan ^{-1}\left(t / r_{c}\right)}{2}\right),
$$

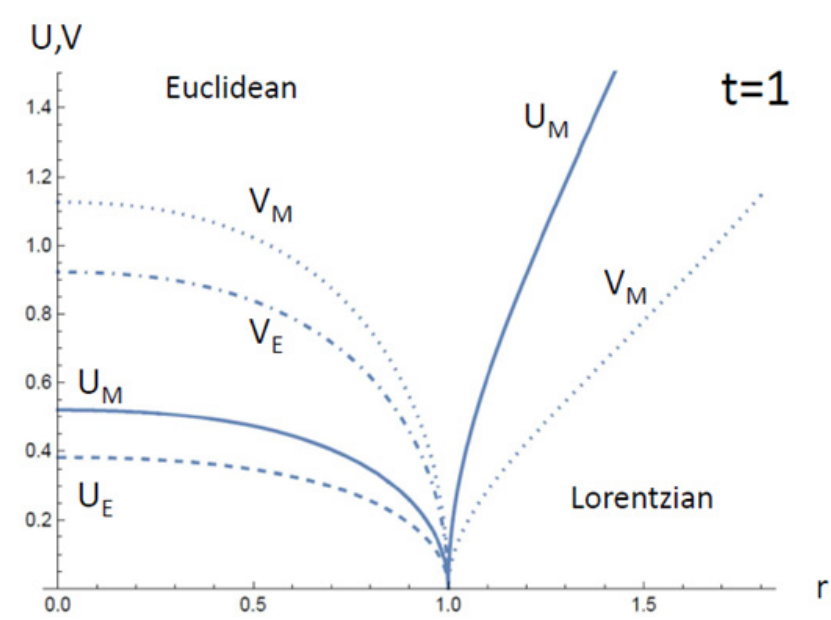

Figure I Two components of (modified) Kruskal-Szekeres coordinates, $U$ and $V$ are shown with $r_{c}=1$ at $t=1$. Solid (dotted) lines show the $U_{\mathrm{M}}-$ ( $V_{\mathrm{M}}-$ ) coordinate, respectively. In the Euclidean-region $r<r_{c}=1, U_{\mathrm{E}}^{\mathrm{M}}$ and $V_{\mathrm{E}}$ are shown by dashed and dot-dashed lines, respectively.

\section{Euclidian-balls and cosmological inflation}

A creation of EBs in the Lorentzian universe may be possible by taking account of a stochastic effect of the general relativity. ${ }^{14}$ Under the stochastic quantization, classical solutions of the general relativity can be obtained by taking an ensemble average of the space time manifold. Ensembles of the space-time manifold can be expressed as $\mathcal{E}=\mathcal{E}_{+} \oplus \mathcal{E}_{-} \oplus \mathcal{E}_{+}$, where $\mathcal{E}_{+}\left(\mathcal{E}_{-}\right)$is a set of the Euclidean-(Lorentzian-)space, respectively. A third ensemble $\mathcal{E}_{+}$is a set of Lorentzian-manifolds in which the Euclidean-manifold is

$$
V_{\mathrm{E}}=\left(1-\frac{r}{r_{c}}\right)^{1 / 2} e^{r / 2 r_{c}} \cos \left(\frac{\tan ^{-1}\left(t / r_{c}\right)}{2}\right)
$$

which gives a line element as;

$$
-d s_{\mathrm{E}}^{2}=4 \frac{r_{c}^{3}}{r}\left(1+\frac{t^{2}}{r_{c}^{2}}\right) e^{-r / r_{c}}\left(d U_{\mathrm{E}}^{2}+d V_{\mathrm{E}}^{2}\right)+r^{2} d \Omega .
$$

At $r=r_{c}$, both solutions coincide as $\left(U_{\mathrm{M}}, V_{\mathrm{M}}\right)=\left(U_{\mathrm{E}}, V_{\mathrm{E}}\right)=(0,0)$. New coordinates are also continuous in entire region of the space as shown in Figure 1. The Euclidean Kruskal-Szekeres coordinates are similar to the Lorentzian ones at the inside of the EB. The Penrose diagram is shown in Figure 2. A region below a 45 degree line is a Lorentzian solution of a Schwarzschild black hole. A standard transformation from Kruskal-Szekeres coordinates to Penrose one $(U, V)$ is used in this region. In the Euclidean region, we set

$\left(U_{\mathrm{E}}, V_{\mathrm{E}}\right)=(U, V)$. A centre of the $\mathrm{EB}, r=0$, is a circle with unit radius, and thus there are no world lines connected from inside of the EB to the asymptotic observer at $r \rightarrow \infty$. Therefore, the EB cannot be distinguished from a Schwarzschild black hole with a mass $M=r_{c} / 2$.

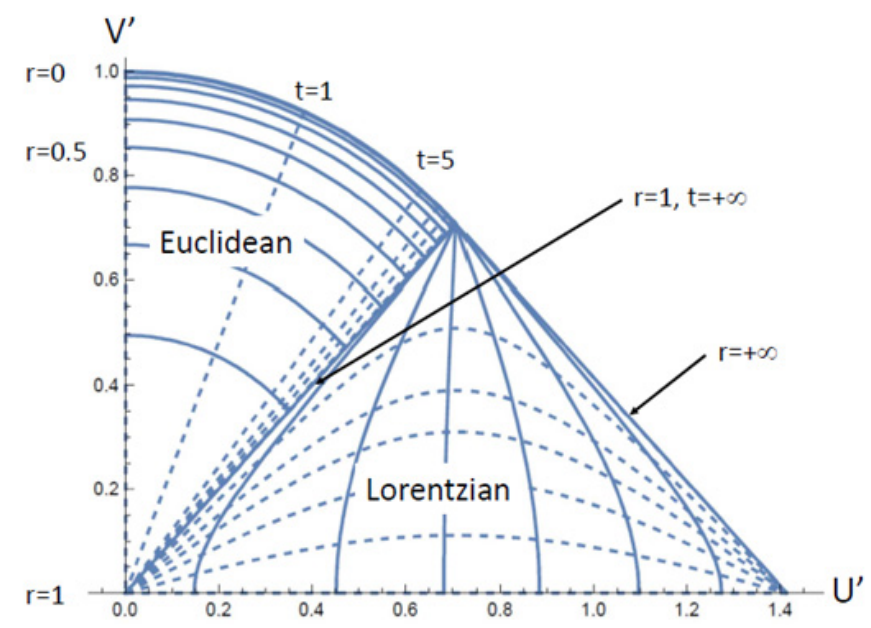

Figure 2 The Penrose diagram for the embedded solution. Solid (dotted) lines show the $r=(t=)$ constant line, respectively. Only a region $t>0$ including an asymptotic observer is drawn in the figure. The 45 degree line shows the event horizon, which is $r=1$ and $t=+\infty$.

embedded, and vice versa. An element of ensembles $\mathcal{U}_{\bullet} \in \mathcal{E}$. is referred to as the "sample universe", and $\mathcal{E}_{+}\left(\mathcal{U}_{+}\right)$are called "chimera ensemble (chimera space)", respectively. An EB-solution in a FLRW universe is an example of the chimera universe. Each element of an ensemble is assumed to be labelled by a order parameter $\tau \in \mathbb{R}$. A distance between two samples $\left\|\mathcal{U}_{\bullet}(\tau)-\mathcal{U}_{\bullet}(\tau)\right\| \geq 0$ is also introduced such as $\lim _{\delta \rightarrow 0}\left\|\mathcal{U}_{\bullet}(\tau)-\mathcal{U}_{\bullet}(\tau+\delta)\right\|=0$. The order parameter is not a component of the space time coordinate (time in a standard sense), but 
an independent parameter from the coordinate, such as the fictitious time in the stochastic quantization by Parisi $\& \mathrm{Wu}^{16}$.

Suppose the space time manifold of the universe was entirely Euclidean before the inflation as discussed by Hawking \& Hartle ${ }^{17}$ and had only one scalar filed in it. This universe is referred to as a pre-universe hereafter. The pre-universe is assumed to be a compact and small. The scalar field played a role to make the pre-universe a compact, and to fix the scale of the pre-universe. (If there is only the space time without any fields, one cannot measure a distance.) The energy momentum tensor of the perfect fluid in a Lorentzian-space is $T_{\mathrm{M}}=\operatorname{diag}\left(c^{2} \rho,-P,-P,-P\right)$, and it in the Euclidean-space is

$T_{\mathrm{E}}=\operatorname{diag}\left(c^{2} \rho, P, P, P\right)$, where $\rho$ and $P$ are a density and pressure of fluid, respectively. Physical constants are written explicitly in this section. From the Friedmann equation for the Lorentzian space, the Hubble parameter $H_{\mathrm{M}}(t)$ follows an equation of;

$$
\frac{d H_{\mathrm{M}}}{d t}:=\dot{H}_{\mathrm{M}}=-4 \pi G\left(1+\frac{P}{\rho c^{2}}\right) \rho+\frac{K c^{2}}{a^{2}},
$$

where $G$ is the Newtonian gravitational constant and $a$ is a scale function of $t$. The curvature is assumed to be positive $K>0$. Conversion of equation (7) from the Lorentzian space to Euclidean space can be done by a simple replacement of $c \rightarrow i c$ as

$$
\frac{d H_{\mathrm{E}}}{d t}:=\dot{H}_{\mathrm{E}}=-4 \pi G\left(1-\frac{P}{\rho c^{2}}\right) \rho-\frac{K c^{2}}{a^{2}} .
$$

For the scalar field, $P /\left(\rho c^{2}\right)=1 / 3$. Therefore, the scalar field in the Euclidean pre-universe induced positive pressure (from the internal observer point of view). During a period sampling the Euclidean-ensemble, the pre-universe stayed in the same scale. After enough duration of $\tau$, the pre-universe is expected to be uniform and isometric, and it must be highly curved due to the pressure of the scalar filed. If the Euclidean ensemble is connected to the chimera ensemble on some region of $\tau$, the pre-universe stared sampling universes in the chimera ensemble in some occasion. In a region of a Lorentzian space in the pre-universe, the scalar field caused an expanding force to the remnant, and then the pre-universe started an explosion inflationary.

On the other hand, the Euclidean region of the pre-universe stayed the same size. A quantitative sketch of a formation of the EBs is as follows (Figure 3): Suppose two Lorentzian space are embedded in the Euclidean space (Figure 3A). Each Lorentzian space had the FLRW metric with a positive curvature and had an event horizon at $r=1 / K^{1 / 2}$. The metric is continuously connected to the Euclidean space at the surfaces. At first distance between two surfaces was much larger than the Planck length. When two surfaces of the Lorentzian space approached within an order of the Planck length, the quantum fluctuation made clacks in the Euclidean-space (Figure 3B) and two Lorentzian regions merged together. Euclidean spaces embedded in the Lorentzian space became 3-dimensional ball due to the scalar field encapsulated in them (Figure 3C). Therefore EBs is distributed on the boundary of two Lorentzian region (named Via). At the Euclidean region among three or more Lorentzian region (named Piazza), larger EBs can be formed as shown in Figure 3D. When the inflation period was terminated, EBs are distributed at the via (small EBs) and the
Piazza (large EBs). Cosmic voids are corresponding to a central part of the Lorentzian space in the pre-universe just before the inflation. In this scenario, requirements for the $e$-folding due to the inflation may be relaxed compared from the standard scenario, because the preuniverse was already in the thermal equilibrium before the inflation. However the inflation itself is necessary to realize the flat universe form the highly curved pre-universe. After the inflation, the energy of the scalar field in the Lorentzian region is converted to visible matter/ gauge fields during the big bang. On the other hand, the energy stored in the EBs becomes invisible other than the gravitational interaction.

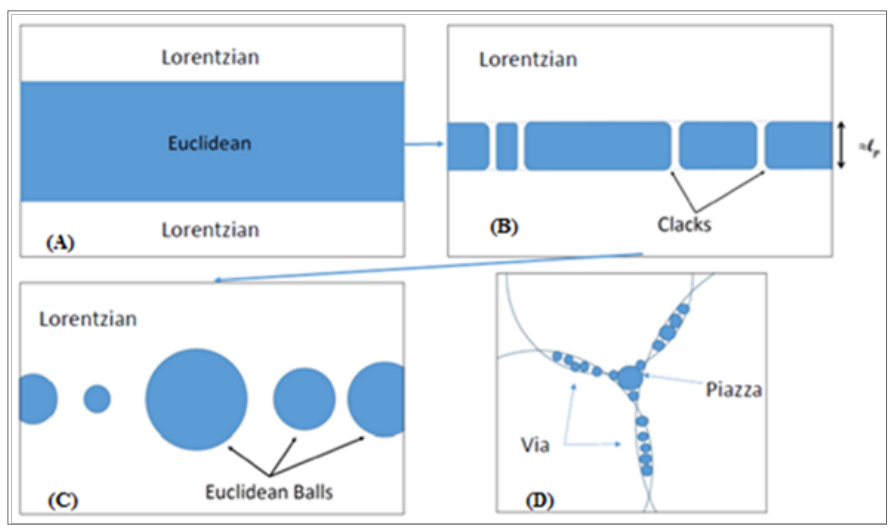

Figure 3 A schematic drawing of a formation of HBs. (A) Close-up view of the Euclidean-space (shadowed area) sandwiched by two (expanding) Lorentzian space. (B) When a distance between two Lorentzian spaces became an order of the Planck length, Lorentzian spaces are bridged due to the quantum fluctuation. (C) EBs are formed on the surface between two Lorentzian spaces (Via) are collide each other. (D) In a region among three or more Lorentzian space (Piazza), lager EBs may be formed compared with those in the Via.

\section{Indirect evidences of euclid-balls}

While the EB is very similar to the primordial black hole, there is critical difference such that the EB cannot emit the Hawking radiation. A reason why the EB does not evaporate is simple: Although a virtual pair of quanta is created at the surface by the quantum effect of the field, none of them can go into the EB because of its metric, and thus it cannot propagate to the asymptotic observer, due to the energy momentum conservation. Therefore, the EB is a stable object and can play a role of the dark matter to contribute a formation of galaxies, and survived until the current universe. A total amount of EBs is free from strong constraints ${ }^{18}$ from astronomical observations. If a fraction of the energy which belongs to the Lorentzian region was $\Omega_{\mathrm{b}} / \Omega_{\mathrm{CDM}}$ ,$^{7,8} \sim 0.0484 / 0.258=0.187$ in the pre-universe, it is conserved until current universe, where $\Omega_{\mathrm{b}}\left(\Omega_{\mathrm{CDM}}\right)$ is a baryon (cold dark matter) density, respectively. Simple consequence of this model is that the dark matter cannot be observed by the detector whose target is weakly interacting particles or decay products of the dark matter, because the EB interacts with visible part of the universe only through the gravitational interaction.

Another consequence is the DM can have a mass spectrum, which means that heavy EBs can be produced as mentioned before. Recent observation reported that the almost all galaxies have a supper massive black hole (SMBH), which have a mass about $10^{6} M_{\odot} \sim 10^{8} M_{\odot}$, at the centre of the galaxy. ${ }^{19,20}$ Several formation mechanisms of such a heavy black hole are proposed, e.g., the SMBH are rapidly grown from the massive star of Pop III type ${ }^{21-23}$ in the early universe, or 
directory formed from dens gases. ${ }^{24-28}$ In both cases, an existence of the seed black hole with a mass about $10 M_{\odot} \sim 100 M_{\odot}$ is necessary to from the SMBH. Moreover, the seed black hole must not be rotating, because an angular momentum disturbs a rapid growing of the black hole. A recent observation ${ }^{29}$ suggests the SMBH is appeared only 900Myr (500Myr) after the big bang (the Pop II, III star formation), respectively. Those observations are contradicted to the standard scenario of the $\Lambda_{\mathrm{CMD}}$ model. ${ }^{30}$ In the EB scenario, EBs may have a mass spectrum from the Planck mass to a certain upper bound. Moreover, EBs is expected to be created without an angular momentum, due to an isometry of the Euclidean-space in the preuniverse. Therefore, the EBs with a large diameter can be a seed of the $\mathrm{SMBH}$. In order to estimate a mass spectrum of the EB created by the inflation of the Lorentzian part of the pre-universe, one has to solve the Einstein equation with a scalar filed in the chimera space, which is beyond the scope of this report.

Even if heavy EBs are formed as much as the stellar black holes, a observation of the EB is more difficult than that of the stellar black hole, because 1) EBs may not have a visible partner who supply hot gasses, and 2) EBs have small angular momentum. A possible method to observe EBs is to use the gravitational wave caused by a fusion of two ESs. There are several gravitational-wave observatories, such as advanced VIRGO, ${ }^{31}$ advanced LIGO, ${ }^{32}$ and KAGRA ${ }^{33}$. A critical (minimum) mass of the stellar black hole is estimated to be $\sim 2 M_{\odot}$ . If gravitational wave observatories will observe gravitational wave from a fusion of two black holes less than the critical mass, it is a candidate of a signal from the EB or primordial black hole. An observation of gravitational wave from merging two neutron stars (GW170817) is reported. ${ }^{34}$ Gravitational wave due to a fusion of binary neutron star can be distinguished from those from black holes (EBs). Another possible signal of gravitational wave from EBs may be hidden in a B-mode polarization of the CMB. EBs existed when the inflation was terminated and stared growing to the SMBH. Fusion of EBs started immediately after the inflation and emitted gravitational wave. This gravitational wave overlaps the primordial gravitational wave $^{2}$ produced during the inflation and put fingerprints on the CMB.

If the EB captured a source of hot gasses, a direct observation of the $\mathrm{EB}$ as the black hole with mass less than the critical mass is also possible. A small radius EBs (smaller than the critical mass) can emit a large total luminosity, because the hot gasses can go to a stronger gravity region than for stellar black holes before reaching a event horizon. The smallest black hole ever observed is XTE J1650500, whose mass is estimated to be $2.7 M_{\odot} \sim 7.3 M_{\odot} \cdot{ }^{36}$

Another candidate to observe the EB is the $\gamma$-ray burst (GRB), especially those with large redshift. Possible aspects of a black hole burst are discussed..$^{37}$ The Swift satellite confirmed eight GRBs with $z>6$ during its ten years operation ${ }^{3}$. A rate of the GRB at $z>6$ is estimated $\sim 0.6$ bursts $\mathrm{yr}^{-1} \mathrm{Sr}^{-1} .^{38}$ There is possibility that a part of those GRBs comes from a fusion of the EB and other stellar objects, such as nuclear star or massive gas stars.

\section{Discussions and Summary}

We introduced the EBs as a new candidate of the dark matter, that is four-dimensional Euclid-space embed in the universe. The EB solution is very similar to a Schwarzschild black hole, and also to

${ }^{2}$ See, for example, a summary report ${ }^{35}$ and references there in.

${ }^{3} \mathrm{~A}$ list of GRBs with $z>6$ and their detailed discussions can be found in Salvaterra R, et al. ${ }^{38}$. the FLRW solution. The FLRW solution is the Lorentzian solution embedded in the Euclidean space, and the EB solution is vice versa. If the pre-universe before the cosmological inflation had the Euclidean metric, ${ }^{14,17}$ EBs can be created during the inflation and stably survived at the current universe. According to the model proposed in this study, the universe can be understood as the chimera space including large amount of EBs in the almost flat Lorentzian space. This model potentially explains an origin of the $\mathrm{DM}$, the homogeneity problem of the universe, formation of the SMBH, and an origin of the GRBs with large redshift.

\section{Acknowledgements}

I appreciate the kind hospitality of all members of the theory group of Nikhef, particularly Prof. J.Vermaseren and Prof. E. Laenen. A major part of this study has been conducted during my stay at Nikhef in 2016 and 2017. In addition, I would like to thank Dr. Y. Sugiyama for his continuous encouragement and fruitful discussion.

\section{Conflicts of interest}

Author declares there is no conflict of interest.

\section{References}

1. Kapteyn JC. First Attempt at a Theory of the Arrangement and Motion of the Sidereal System. Astrophysical Journal. 1922;55:302-328.

2. Drees M, Gerbier G. Darm matter (Review of Particle Physics). Chinese Physics C. 2016;40(10):1-10.

3. Bertone G. Particle Dark Matter: Observations, Models and Searches. UK: Cambridge University Press; 2010.

4. Bertone G, Hooper H, Silk J. Particle dark matter: evidence, candidates and constraints. Physics Reports. 2005;405(5-6):279-390.

5. Kolb EK, Turner MS. The Early Universe. Frontiers of Physics. 1990;69:1-547.

6. Tyson JA, Wenk RA, Valdes F. Detection of systematic gravitational lens galaxy image alignments-Mapping dark matter in galaxy clusters. Astrophysical Journal. 1990;349:L1-L4.

7. Ade PAR, Aghanim N, Arnaud M, et al. Planck 2015 results. XIII. Cosmological parameters. Astronomy \& Astrophysics. 2016;594(A13).

8. Patrignani C, Agashe K, Aielli G, et al. Review of Particle Physics. Chinese Physics C. 2016;40(10):1-10.

9. Peccei RD, Quinn HR. CP Conservation in the Presence of Instantons. Physical Review Letters. 1977;38(25):1440-1443.

10. Peccei RD, Quinn HR. Constraints Imposed by CP Conservation in the Presence of Instantons. Physical Review D. 1977;16(6):1791-1797.

11. Kohri K, Lyth D, Melchiorri A. Black hole formation and slow-roll ination. Journal of Cosmologyand Astroparticle Physics. 2008;(4):1-21.

12. Olive KA. Supersymmetric Dark Matter after Run I at the LHC: From a $\mathrm{TeV}$ to a PeV. USA: Cornell University Library; 2015. p. 1-22.

13. Bueno Sanchez JC, Bastero-Gil M, Berera A, et al. The gravitino problem in supersymmetric warm ination. Journal of Cosmology and Astroparticle Physics. 2011. 1103 p.

14. Kurihara Y. Stochastic metric space and quantum mechanics. Journal of Physics Communications. 2018;2(3):1-33.

15. Weinberg S. Gravitation and cosmology: principles and applications of the general theory of relativity. USA: John Wiley \& Sons; 1972. 
16. Parisi G, Wu YS. Perturbation theory without gauge fixing. Scientia Sinica. 1981;24(4):483-469.

17. Hartle JB, Hawking SW. Path-integral derivation of black-hole radiance. Physical Review D. 1976;13(8):2188-2203.

18. Carr BJ, Kohri K, Sendouda Y, et al. Constraints on primordial black holes from the Galactic gamma-ray background. Physical Review D. 2016;94(4).

19. Urry CM, Padovani P. Unified schemes for radio-loud active galactic nuclei. Publications of the Astronomical Society of the Pacific. 1995;107(715).

20. Antonucci R. Unified models for active galactic nuclei and quasars. Annual Review of Astronomy and Astrophysics. 1993;31(1):473-521.

21. Volonteri M, Haardt F, Madau P. The assembly and merging history of supermassive black holesin hierarchical models of galaxy formation. The Astrophysical Journal. 2003;582(2):559-573.

22. Volonteri M, Madau P, Quataert E, et al. The distribution and cosmic evolution of massive black hole spins. The Astrophysical Journal. $2005 ; 620(1) ; 69-77$.

23. Volonteri M, Salvaterra R, Haardt F. Constraints on the Accretion History of Massive Black Holes from Faint X-ray Counts. Monthly Notices of the Royal Astronomical Society. 2006;373(1):121-127.

24. Cattaneo A, Haehnelt MG, Rees MJR. The distribution of supermassive black holes in the nuclei of nearby galaxies. Monthly Notices of the Royal Astronomical Society. 1999;308(1):77-81.

25. Umemura M, Loeb A, Turner EL. Early Cosmic Formation of Massive Black Holes. Astrophysical Journal. 1993;419.

26. Loeb A, Rasio FA. Collapse of primordial gas clouds and the formation of quasar black holes. Astrophysical Journal. 1994;432(1):52-61.

27. Eisenstein DJ, Loeb A. Origin of quasar progenitors from the collapse of low-spin cosmological perturbations. Astrophysical Journal. 1995;443(1):11-17.
28. Koushiappas SM, Bullock JS, Dekel A. Massive black hole seeds from low angular momentum material. Monthly Notices of the Royal Astronomical Society. 2004;354(1):292-304.

29. Wu XB, Wang F, Fan X, et al. An ultraluminous quasar with a twelvebillion-solar-mass black hole at redshift 6.30. Nature. 2015;518:512515 .

30. Melia F, McClintock TM. Supermassive Black Holes in the Early Universe. Proceedings of the Royal Society A: Mathematical, Physical and Engineering Science. 2015;471(2184).

31. Abbott BP, et al. GW150914: The Advanced LIGO Detectors in the Era of First Discoveries. Physical Review Letters. 2016;116(13):1-12.

32. Zevin M, Coughlin S, Bahaadini S, et al. Gravity Spy: Integrating Advanced LIGO Detector Characterization, Machine Learning, and Citizen Sciencear. USA: Cornell University Library; 2016. p. 1-27.

33. Somiya K. Detector configuration of KAGRA: The Japanese cryogenic gravitational-wave detector. Classical and Quantum Gravity. 2012;29(12).

34. Abbott BP, Abbott R, Abbott TD, et al. Gw170817: Observation of gravitational waves from a binary neutron star inspiral. Physical Review Letters. 2017;119(16).

35. Kamionkowski M, Kovetz ED. The Quest for B Modes from Inationary Gravitational Wavesar. USA: Cornell University Library; 2015. p. 1-45.

36. Shaposhnikov N, Titarchuk L. Determination of black hole masses in galactic black hole binaries using scaling of spectral and variability characteristics. The Astrophysical Journal. 2009;699(1):453-468.

37. MacGibbon JH, Carr BJ, Page DN. Do evaporating black holes form photospheres? Physical Review D. 2008;78(6).

38. Salvaterra R. High redshift Gamma-Ray Bursts. Journal of High Energy Astrophysics. 2015;7:35-43. 